\title{
Iterative CT reconstruction using shearlet-based regularization
}

\author{
Bert Vandeghinste $^{a}$, Bart Goossens ${ }^{b}$, Roel Van Holen ${ }^{a}$, Christian Vanhove ${ }^{a}$, Aleksandra \\ Pižurica $^{b}$, Stefaan Vandenberghe ${ }^{a}$ and Steven Staelens ${ }^{a c}$ \\ ${ }^{a}$ MEDISIP research group, Ghent University-IBBT, 9000 Gent, Belgium; \\ ${ }^{b}$ IPI research group, Ghent University-IBBT, 9000 Gent, Belgium; \\ ${ }^{c}$ Molecular Imaging Centre Antwerp, University of Antwerp, 2650 Edegem, Belgium
}

\begin{abstract}
In computerized tomography, it is important to reduce the image noise without increasing the acquisition dose. Extensive research has been done into total variation minimization for image denoising and sparse-view reconstruction. However, TV minimization methods show superior denoising performance for simple images (with little texture), but result in texture information loss when applied to more complex images. Since in medical imaging, we are often confronted with textured images, it might not be beneficial to use TV. Our objective is to find a regularization term outperforming TV for sparse-view reconstruction and image denoising in general. A recent efficient solver was developed for convex problems, based on a split-Bregman approach, able to incorporate regularization terms different from TV. In this work, a proof-of-concept study demonstrates the usage of the discrete shearlet transform as a sparsifying transform within this solver for CT reconstructions. In particular, the regularization term is the $\ell_{1}$-norm of the shearlet coefficients. We compared our newly developed shearlet approach to traditional TV on both sparse-view and on low-count simulated and measured preclinical data. Shearlet-based regularization does not outperform TV-based regularization for all datasets. Reconstructed images exhibit small aliasing artifacts in sparse-view reconstruction problems, but show no staircasing effect. This results in a slightly higher resolution than with TV-based regularization.
\end{abstract}

Keywords: Noise regularization, Shearlets, CT regularization, Shearlet-based regularization, Total Variation

\section{INTRODUCTION}

In the field of in-vivo micro computerized tomography $(\mu \mathrm{CT})$, it is important to reduce image noise. It is impossible to solve this issue with longer acquisition times because of limitations on administered dose and anesthetics. Furthermore, the recently emerged research topics on multi-energy or spectral energy imaging could potentially increase the soft tissue contrast by weighting the x-ray photons according to their energy. However, the material decomposition methods are highly susceptible to high noise, and additional noise will be introduced due to extra binning. Hence, it is important to reduce the image noise through advanced reconstruction techniques. This may provide us with the perfect tools to lower $\mu \mathrm{CT}$ radiation dose in general, make in-vivo spectral $\mu \mathrm{CT}$ possible, and ultimately obtain increased soft tissue contrast.

Total variation (TV) minimization is one of the techniques that have been extensively investigated in the last decade in the context of image denoising and sparse-view reconstruction. ${ }^{1-4}$ It is generally accepted that these TV-based methods have superior denoising performance when applied to simple classes of images with no texture, such as images of conic shapes with flat colors. However, these methods often produce cartoon-like approximations when applied to images that contain complex textures and shading. This distortion makes the TV regularizer less suitable for medical images with complex textures, including some types of CT images. ${ }^{2}$ Consequently, it could be beneficial to use regularizers other than TV.

It has been suggested ${ }^{5}$ to use the $\ell_{1}$-norm of the simplest possible wavelet, the Haar transform, instead of TV. This has already been investigated for regularized CT reconstruction by Garduno et al. ${ }^{6}$ However, they

Further author information: (Send correspondence to Bert Vandeghinste)

E-mail: Bert.Vandeghinste@ugent.be, Telephone: +3293324322 
found that reconstructions with a small $\ell_{1}$-norm of its Haar transform are not more effective and are not anymore useful from the medical diagnostic point of view than reconstructions that have a small TV value. The authors did not explain this result. Thus, the search for an objective function that provides diagnostically efficacious reconstruction from a limited number of CT projections remains open. ${ }^{6}$

Previously, we proposed a general regularization method, based on an augmented Lagrangian (or splitBregman) approach, ${ }^{7-9}$ as an alternative method for sparse-view CT reconstruction. This very general method allows the incorporation of extra regularization terms quite easily. In the work presented here, we investigate if using shearlets ${ }^{10,11}$ in regularized CT reconstruction is more efficient than using TV. In theory, shearlets have better directional sensitivity, better $\ell_{1}$-norm sparsity and, because of a different underlying image model, do not lead to the staircasing effect.

The remainder of this paper is organized as follows. In Sec. 2.2, we introduce the discrete shearlet transform, the problem formulation and the mathematical background for using the split-Bregman approach in CT. Section 3 describes the set-up for the evaluation on simulated and measured preclinical $\mu \mathrm{CT}$ data. In Sec. 4 we compare the reconstruction performance of shearlet-based regularization to TV-based regularization, in context of lowcount and sparse-view reconstruction. These results are then discussed in Sec. 5, where suggestions are also made for further research. Our conclusions are in Sec. 6 .

\section{PRELIMINARIES}

\subsection{The shearlet transformation}

The wavelet transform decomposes an image by projecting onto several dilated and translated version of one single function, namely the mother wavelet. It unfortunately has a very limited ability to resolve edges and other distributed discontinuities which usually occur in multidimensional data. These properties make the wavelet transform not suitable for CT reconstruction.

The shearlet transform ${ }^{10,11}$ is a non-isotropic version of the wavelet transform. It was shown ${ }^{11}$ that the asymptotic decay rate of the continuous shearlet transform, for fine scales, can be used to find both the location and the orientation of the edges of an image, and that the coefficients of large magnitude will come from edges. Furthermore, the decay rate across scales can be used to distinguish between noise spikes and edges.

These properties theoretically make shearlets a better choice for use in regularized CT reconstruction than TV. TV only takes the horizontal and vertical directions into account at one single resolution, while shearlets will take edges into account in a multitude of directions, at different scales. By setting shearlet coefficients below a certain threshold to zero, one is also able to extract the features of the image above a certain scale. ${ }^{12}$ Additionally, in compressed sensing the goal is to use a transformation with the best approximation (compaction) properties. In higher dimensions, shearlets are a better candidate for this than wavelets or TV.

A discrete version of the shearlet transformation has previously been implemented by Goossens et al. ${ }^{13}$ based on the Meyer wavelet. This novel design has a very low redundancy factor and a relatively short computing time compared to other implementations.

\subsection{Split-Bregman framework}

Previously, we have developed the split-Bregman framework for regularized CT reconstruction. ${ }^{8,9}$ We denote an $\ell_{1}$-norm by $|\cdot|_{1}$ and an $\ell_{2}$-norm by $\|\cdot\|_{2}$. The following minimization problem is solved:

$$
\hat{\mathbf{x}}=\underset{\mathbf{x}}{\arg \min }|\Phi(\mathbf{x})|_{1}+\lambda\left\|\mathbf{C}^{-1 / 2}(\mathbf{y}-\mathbf{W} \mathbf{x})\right\|_{2}^{2},
$$

with $\mathbf{x}$ the unknown reconstructed image, $\Phi$ a linear sparsifying transformation (such as the discrete gradient transformations, ${ }^{14}$ or the shearlet transformation), $\lambda$ a constant which determines the amount of data-fitting and amount of regularization, $\mathbf{C}$ a pre-whitening term, $\mathbf{y}$ the measured data and $\mathbf{W}$ the system matrix. This cost function can be minimized by iterating over the following three equations: 


$$
\begin{aligned}
& \mathbf{x}^{(i+1)}=\underset{\mathbf{x}}{\arg \min } \frac{\lambda}{2}\left\|\mathbf{C}^{-1 / 2}(\mathbf{y}-\mathbf{W} \mathbf{x})\right\|_{2}^{2}+\frac{\mu}{2}\left\|\mathbf{d}^{(i)}-\Phi(\mathbf{x})-\mathbf{b}^{(i)}\right\|_{2}^{2} \\
& \mathbf{d}^{(i+1)}=\underset{\mathbf{d}}{\arg \min }|\mathbf{d}|_{1}+\frac{\mu}{2}\left\|\mathbf{d}-\Phi\left(\mathbf{x}^{(i+1)}\right)-\mathbf{b}^{(i)}\right\|_{2}^{2} \\
& \mathbf{b}^{(i+1)}=\mathbf{b}^{(i)}+\left(\Phi\left(\mathbf{x}^{(i+1)}\right)-\mathbf{d}^{(i+1)}\right) .
\end{aligned}
$$

The quadratic $\ell_{1}$ - and the $\ell_{2}$-norm from Eq. 1 are now split into different minimization problems. Variables $\mathbf{d}^{(0)}$ and $\mathbf{b}^{(0)}$ are initialized with $\mathbf{0}$. Constant $\mu$ determines the convergency speed through the ratio $\mu / \lambda$. For a more detailed mathematical derivation, the reader is referred to Vandeghinste et al. ${ }^{9}$

\section{MATERIALS AND METHODS}

\subsection{Simulated data}

Fan-beam data was simulated using MC-GPU v1.215*, a GPU-accelerated x-ray transport simulator. The 4D Mouse Whole Body (MOBY) ${ }^{16}$ phantom was used to generate a voxelized mouse phantom with dummy organ activities. Each specific activity value was then related to different materials, used to index material tables in MC-GPU. The detector was simulated as a perfect detector with $100 \%$ efficiency, and consists of 296 elements with a pixel pitch of $0.145 \mathrm{~mm}$, acquiring 360 uniformly spaced projection views over $2 \pi$. All data was generated using a $60 \mathrm{keV}$ monoenergetic x-ray source. The sinogram includes scattered photons.

MC-GPU was used to simulate two datasets, one with $10^{10}$ photons, and one with $10^{6}$ photons per ray. The first dataset was later reduced to only 72 projection views in post-processing. This results in three distinct datasets in total, enabling the evaluation of sparse-view reconstruction and low-count acquisition. The MOBY phantom can then serve as an ideal reference to both.

\subsection{Experimental data}

The X-O CT system (Gamma Medica-Ideas, Northridge, California, USA) was used to obtain preclinical data. This flat-panel cone-beam system consists of a $1280 \times 1120$ detector with a $100 \mu m$ pixel pitch. The tube current is determined automatically during calibration to ensure that the dynamic range of the detector is optimally used. Fan-beam data were generated by retaining only the central detector row. 2048 projection views were obtained over $2 \pi$. Two datasets were generated from this projection data, by removing all but every 8 th projection (256 views) and all but every 16 th projection (128 views).

\subsection{Data reconstruction and analysis}

All datasets were reconstructed using the Simultaneous Iterative Reconstruction Technique (SIRT), split-Bregman using anisotropic TV (SpBR-TV) and split-Bregman using shearlets (SpBR-SH), to a $256^{2}$-grid with $0.13 \mathrm{~mm}$ voxel pitch. In the case of the simulated CT data, the diagonal elements of $\mathbf{C}$ (see Eq. 1) were set to $c_{i i}=e^{-y_{i}}{ }^{17}$ with $y_{i}$ the detected number of counts from the $10^{10}$ photons simulation. As this ground truth data was unavailable for the measured preclinical data, we used the projection data itself as an estimator of $y_{i}$.

For each regularized reconstruction, parameter $\lambda$ was empirically chosen, by reconstructing with different $\lambda$ values and gradually making the search interval smaller, fine tuning the amount of denoising. This generally results in 5 to 10 reconstructions needed to determine a good $\lambda$ value. The parameter $\mu$ was always set to $0.585 \times \lambda$, which was empirically determined.

All SIRT reconstructions converged and were stopped when $\left\|\mathbf{x}^{(i+1)}-\mathbf{x}^{(i)}\right\|_{2}^{2} /\left\|\mathbf{x}^{(i+1)}\right\|_{2}^{2}<10^{-4}$. The SpBRbased reconstructions converged and were stopped at iteration 20.

For data analysis purposes we compared the different reconstructions visually as well as calculating the peak signal-to-noise ratio (PSNR). As reference image for the simulated data the original MOBY phantom image was used, after converting it to attenuation values at $60 \mathrm{keV}$. For the preclinical data, we used the SIRT reconstruction of 2048 projection views.

\footnotetext{
${ }^{*}$ Freely available from http://code.google.com/p/mcgpu/
} 

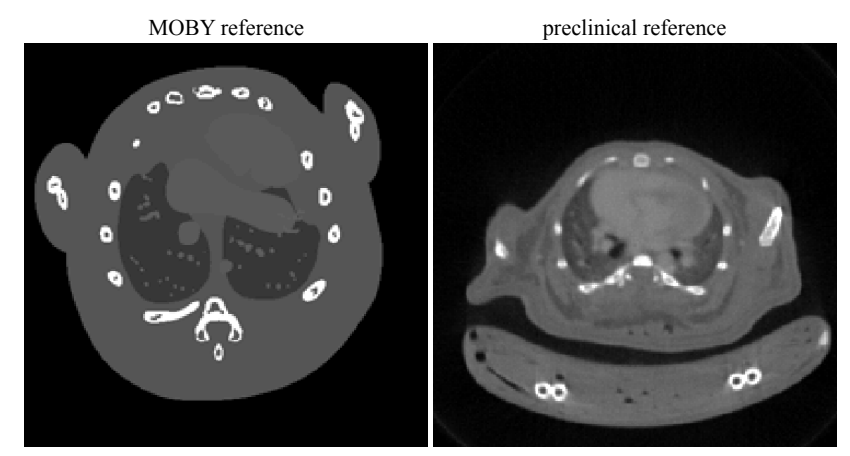

Figure 1. Reference images for the simulated data (MOBY phantom converted to $60 \mathrm{keV}$ attenuation values) and the preclinical data (converged SIRT reconstruction of 2048 projection views).

\section{RESULTS}

We previously developed reconstruction software that can use different regularizers, such as isotropic or anisotropic $\mathrm{TV}$, the discrete wavelet transform or the shearlet transform. ${ }^{9}$ In the present study, we investigated if shearlets have a significant advantage over the common TV norm. We investigated this for two different settings: sparse-view sampling and low-count acquisition.

Figure 1 presents the reference images which will be used in the PSNR comparisons. The MOBY phantom represents bones, lungs, vessels and the heart in one slice, all embedded in soft tissue. This phantom is clearly piecewise constant, as organs are not represented as mixtures of materials. Conversely, the preclinical reference shows both muscle and fat, together with the heart, lungs, bones, vessels and a carbon small animal bed containing copper heating wires.

Figure 2 compares shearlet-based reconstruction to TV-based reconstruction for simulated MOBY data. Both regularizing approaches obtain a higher PSNR than SIRT reconstruction, with SpBR-TV reaching a higher PSNR than SpBR-SH. SpBR-SH shows a small amount of aliasing artifacts due to the low number of views, although it exhibits a higher resolution on the bones compared to SIRT. There is a small Gibbs effect noticeable next to all edges.

In low-count data acquisition, both SpBR methods achieve a significantly higher PSNR than the conventional SIRT reconstruction. SpBR-TV demonstrates patchy behavior, which is also apparent with SpBR-SH, though with a different texture.

Figure 3 shows the reconstructions from the acquired preclinical data. It is apparent from the SIRT reconstructions that the number of projection views determines the amount of noise in the images, but that 256 views is not low enough to get aliasing artifacts similar to those seen in Fig. 2. When 256 views are used (top row), aliasing effects are already visible on the body contour. SpBR-SH has a smoother contour than SpBR-TV. Furthermore, some patchy behavior can be seen when TV is used, especially apparent on the edges between muscle and fat tissue. This effect is not visible with the shearlet-based reconstruction.

When only 128 views are used, aliasing artifacts become visible when SIRT is used, which are not completely removed with SpBR-SH, although the noise is significantly reduced. The SpBR-TV reconstruction shows these aliasing artifacts only slightly. Some staircasing effect is visible with TV, for example on the top edge of the small animal bed. This staircasing leads to combining three seperate vessels into one big vessel just below the lower left corner of the heart, while these three vessels are slightly better delineated in SpBR-SH.

\section{DISCUSSION}

The results obtained are inconclusive to show if shearlets outperform TV regularization in CT reconstruction. Although shearlets do not show any form of piecewise-constant behavior, which is an advantage over TV minimization, they do not tend to approximate uniform regions as well as TV.

Unfortunately, it is very difficult to quantitatively evaluate the usefulness from the medical diagnostic point of view, as a ground truth reference image will be needed. The most-used phantom in CT reconstruction evaluation 


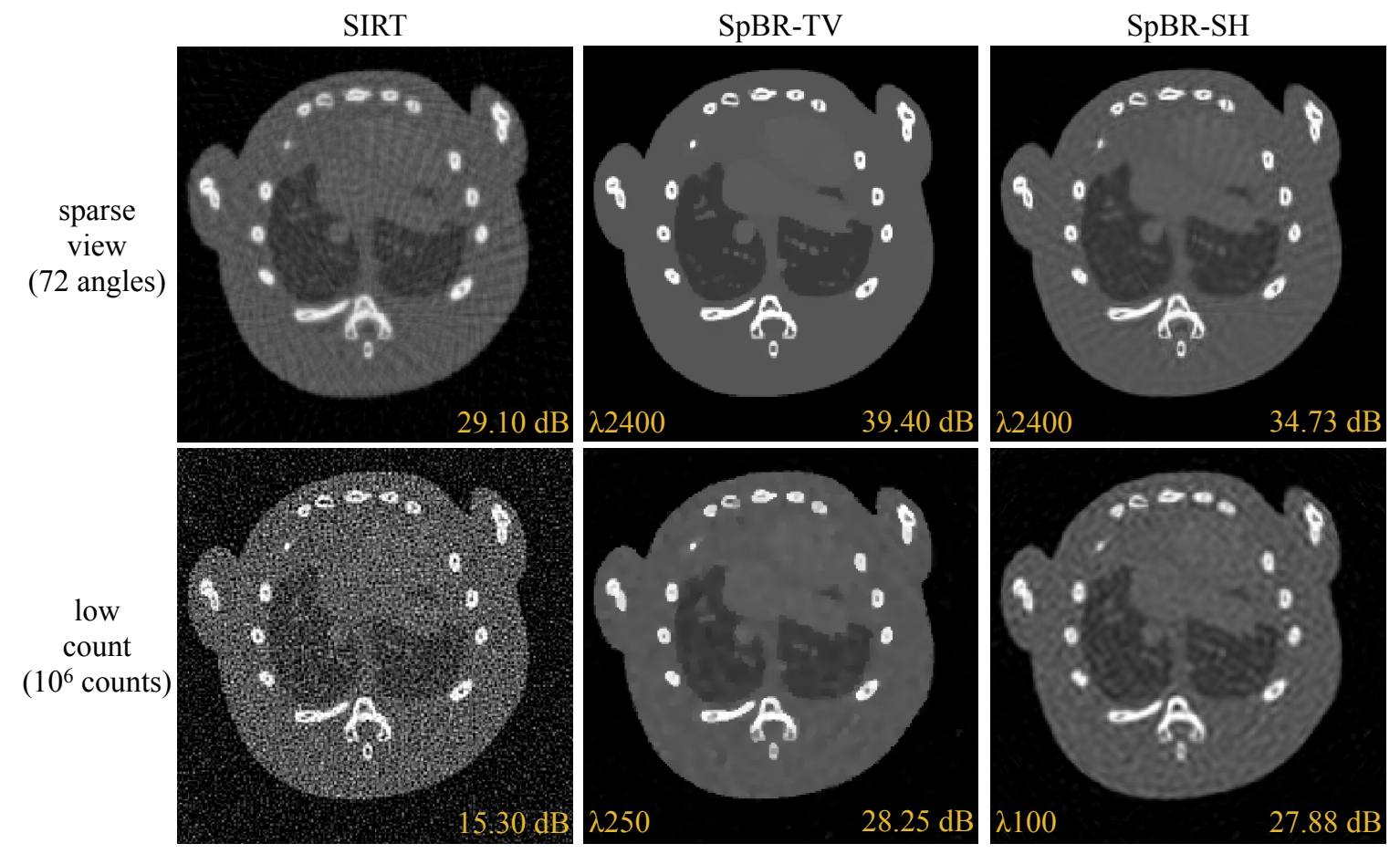

Figure 2. Comparison of SIRT, SpBR-TV and SpBR-SH on simulated MOBY data. Top row: $10^{10}$ counts per ray, 72 projection views. Bottom row: $10^{6}$ counts per ray, 360 projection views. Used $\lambda$ parameter plotted in bottom left. PSNR compared to MOBY phantom, plotted in bottom right.

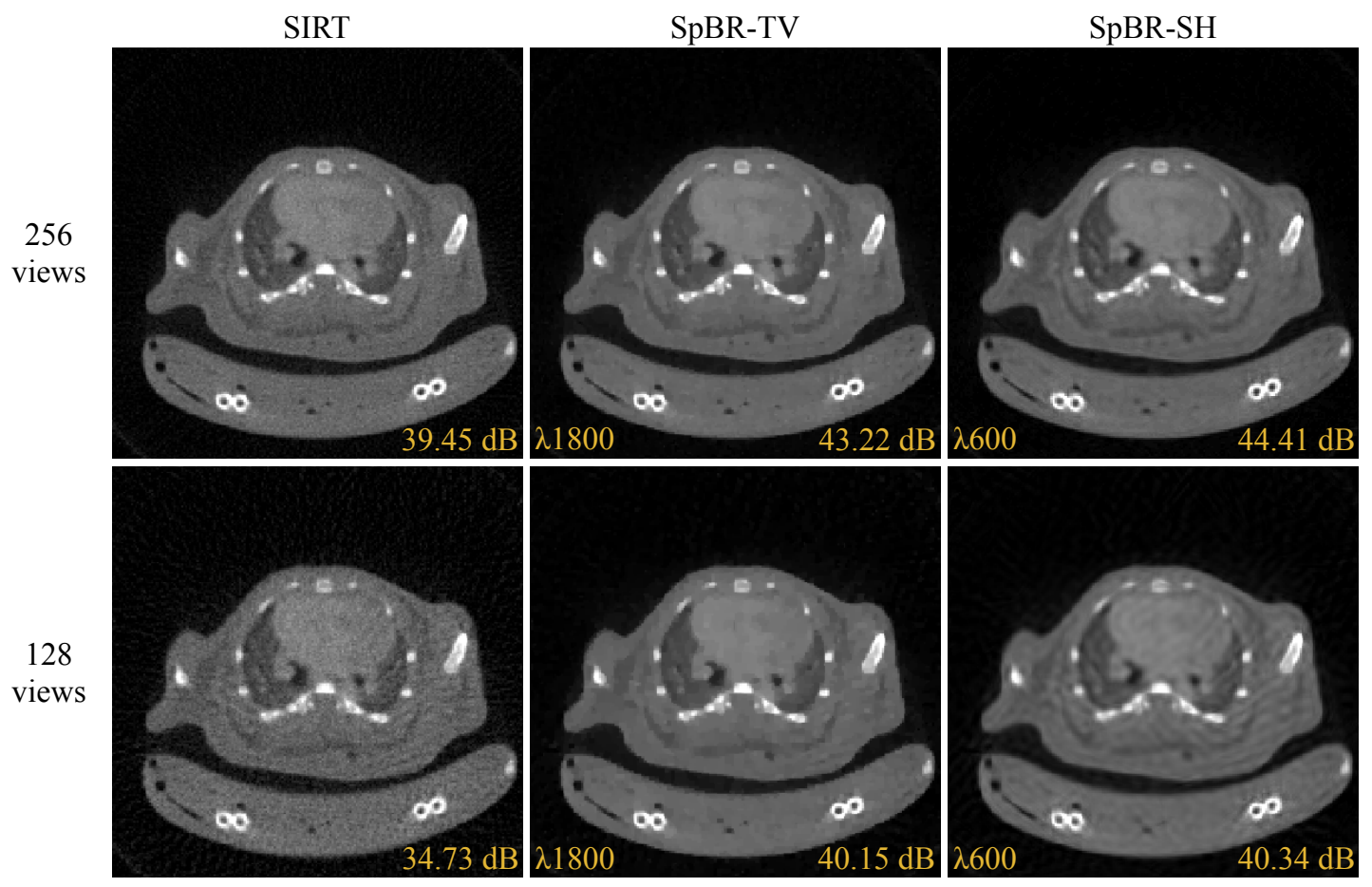

Figure 3. Comparison of SIRT, SpBR-TV and SpBR-SH on acquired preclinical data. Top row: 256 projection views. Bottom row: 128 projection views. PSNR compared to the SIRT reconstruction using 2048 projection views, plotted in bottom right. 
is the Shepp-Logan phantom. It can be defined by adding 10 uniformly-filled ellipses, ${ }^{18}$ and is thus inherently piecewise constant. More realistic simulators and phantoms require a list of materials indexed by a discrete set of voxels. Although this approach is an improvement on the usage of simple phantoms, it still exhibits piecewise constant behavior. This was clearly seen on Fig. 2, where this piecewise constant model is a perfect match for the underlying model of TV. The streaking artifacts can not be efficiently minimized by SpBR-SH, as shearlets maximally correlate with these lines.

A solution to the problem of unrealistically simple phantoms could be to simulate image structures significantly smaller than the system resolution, hereby inducing the partial volume effect during reconstruction. This will effectively result in non-piecewise constant behavior, which should be approximated more accurately by shearlets than with TV. However, we did not yet pursue this further and will do this in the future.

On the other hand, a difference is visible in acquired preclinical data. The reconstructions using shearlets have a slightly higher resolution than TV-based reconstructions, but do not show the patchy behavior of TV, approximating smooth edges with staircase-like figures. The difficulty in this evaluation however is that it is highly subjective, as no true reference image can be constructed. We have used a high-dose acquisition as reference, but this reference image still contains some noise, as can be noticed from Fig. 1. It is thus not a reference image in the strict sense.

In the future, we will investigate if a combination of shearlets with TV provide better diagnostic quality than shearlets or TV in itself. This combination has already been shown effective in Magnetic Resonance Imaging reconstruction. ${ }^{19-21}$ Ideally, we would want to use shearlets for denoising, and TV to minimize the remaining artifacts such as streaking lines and the Gibbs effect.

\section{CONCLUSION}

We have shown that the split-Bregman framework can be used with shearlet regularization to perform iterative CT reconstruction. Shearlets lead to different artifacts than TV, due to a different underlying image model. We were unable to confirm if shearlets are superior over TV for CT regularization, although a subjective comparison suggests that a combination of both might be ideal.

\section{ACKNOWLEDGMENTS}

This work was supported by a PhD grant to Bert Vandeghinste of the Institute for the Promotion of Innovation through Science and Technology in Flanders (IWT-Vlaanderen). Bart Goossens and Roel Van Holen are postdoctoral fellows of the Research Foundation Flanders (FWO). Christian Vanhove is supported by the GROUP-ID consortium.

\section{REFERENCES}

[1] Rudin, L., Ösher, S., and Fatemi, E., "Nonlinear total variation based noise removal algorithms," Physica D. 60, 259-268 (1992).

[2] Herman, G. and Davidi, R., "Image reconstruction from a small number of projections," Inverse Problems 24(4), 045001 (2008).

[3] Lustig, M., Donoho, D., and Pauly, J., "Sparse MRI: The application of compressed sensing for rapid MR imaging," Magnetic Resonance in Medicine 58(6), 1182-1195 (2007).

[4] Defrise, M., Vanhove, C., and Liu, X., "An algorithm for total variation regularization in high-dimensional linear problems," Inverse Problems 27, 065002 (2011).

[5] Chen, G.-H., Tang, J., Nett, B., Qi, Z., Leng, S., and Szczykutowicz, T., "Prior image constrained compressed sensing (PICCS) and applications in x-ray computed tomography," Curr. Med. Imaging. Rev. 6, 119-34 (2010).

[6] Garduño, E., Herman, G., and Davidi, R., "Reconstruction from a few projections by L1-minimization of the Haar transform," Inverse Problems 27(5), 055006 (2011).

[7] Tai, X. and Wu, C., "Augmented lagrangian method, dual methods and split bregman iteration for ROF model," Scale space and variational methods in computer vision 5567, 502-513 (2009). 
[8] Goldstein, T. and Osher, S., "The split bregman method for L1 regularized problems," SIAM J. Imag. Sci. 2(2), 323-343 (2009).

[9] Vandeghinste, B., Goossens, B., De Beenhouwer, J., Pižurica, A., Philips, W., Vandenberghe, S., and Staelens, S., "Split-bregman-based sparse-view CT reconstruction," Proc. Intl. Mtg. on Fully 3D Image Recon. in Rad. and Nuc. Med, 431-434 (2011).

[10] Kutyniok, G. and Labate, D., "Resolution of the wavefront set using continuous shearlets," Trans. Amer. Math. Soc. 361(5), 2719-2754 (2008).

[11] Guo, K., Labate, D., and Lim, W., "Edge analysis and identification using the continuous shearlet transform," Appl. Comput. Harmon. A. 27(1), 24-46 (2009).

[12] Easley, G., Labate, D., and Colonna, F., "Shearlet based total variation for denoising," IEEE Trans. Imag. Process. 18(2), 260-268 (2009).

[13] Goossens, B., Aelterman, J., Luong, H., Pižurica, A., and Philips, W., "Efficient design of a low redundant discrete shearlet transform," Proc. of the 2009 Int. Workshop on Local and Non-Local approximation in Image Processing, Tuusula, Finland, 112-124 (2009).

[14] Yu, H. and Wang, G., "SART-Type Image Reconstruction from a Limited Number of Projections with the Sparsity Constraint," International Journal of Biomedical Imaging 2010, 9 (2010).

[15] Badal, A. and Badano, A., "Accelerating Monte Carlo simulations of photon transport in a voxelized geometry using a massively parallel graphics processing unit," Med. Phys. 36(11), 4878-4880 (2009).

[16] Segars, W., Tsui, B., Frey, E., Johnson, G., and Berr, S., "Development of a 4-D digital mouse phantom for molecular imaging research," Mol. Imaging Biol. 6(3), 149-159 (2004).

[17] Ramani, S. and Fessler, J., "A splitting-based iterative algorithm for accelerated statistical x-ray CT reconstruction," IEEE. Trans. Med. Imag. (2011).

[18] Shepp, L. and Logan, B., "The fourier reconstruction of a head section," IEEE Trans. Nucl. Sci. 21(3), 21-43 (1974).

[19] Pižurica, A., Aelterman, J., Bai, F., Vanloocke, S., Luong, H., Goossens, B., and Philips, W., "On structured sparsity and selected applications in tomographic imaging," SPIE Conference Wavelets and Sparsity XIV, 81381D (2011).

[20] He, L., Chang, T.-C., Osher, S., Fang, T., and Speier, P., "MR image reconstruction from undersampled data by using the iterative refinement procedure," Proc. Appl. Math. and Mech. 7, 1011207-1011208 (2007).

[21] Huang, J., Zhang, T., Li, H., and Metaxas, D., "Composite splitting algorithms for convex optimization," Computer Vision and Image Understanding (2011). 